\title{
Radiation-induced Back Ulcers are Often Overlooked
}

\author{
Young-Don Yoon, Sik Namgoong, Seong-Ho Jeong, Deok-Woo Kim, Eun-Sang Dhong, Seung-Kyu Han \\ Department of Plastic Surgery, Korea University College of Medicine, Seoul, Korea
}

\begin{abstract}
Background: Given that fluoroscopic procedures have been more commonly used, many radiation-induced complications following these radiological interventional procedures should be given attention. However, there are few studies related to radiation-induced ulcers in Korea. Thus, this study aimed to present several cases of radiation-induced ulcers and to investigate the characteristics of radiation-induced ulcers.

Methods: A retrospective review was performed on the maintained databases at our institution. All patients who visited for radiation-induced ulcer treatment between 2010 and 2015 were included in the analysis. We reviewed medical charts and operative data, including patient demographics, photographs, surgical techniques, and postoperative outcomes.

Results: In total, five patients with radiation-induced back ulcers were included and they underwent intravenous antibiotic treatment, conservative dressing therapy, and surgical procedures (e.g., surgical debridement, split-thickness skin graft, and local flap). At a mean follow-up of 11 months, all lesions in the patients healed completely without any recurrence.

Conclusion: Radiation-induced back ulcers are easily overlooked. Thus, accurate assessment and multidisciplinary approaches for treatment of such injuries are crucial.
\end{abstract}

Keywords: Radiation, Fluoroscopic procedure, Back ulcer

\section{Introduction}

Fluoroscopic interventions include many diagnostic studies and therapeutic interventions, such as cardiac angioplasty, coronary stent insertion, and hepatic radiofrequency ablation [1]. Both the radiologists and the patients are exposed to high doses of radiation during interventional procedures. However, many radiologists have focused only on protecting the staff, which are constantly exposed to cumulative radiation [2]. Unfortunately, many physicians are not aware of the skin injuries associated with these procedures that can occur to patients. Therefore, skin lesions associated with fluoroscopic procedures are thought to be underreported [3]. This problem requires attention, especially now that fluoroscopic interventions are frequently performed $[3,4]$. There are several studies that have reported the skin injuries following fluoroscopic procedures. Wagner et al. and Dandurand et al. warned of the risk of radiationinduced ulcers that developed after radiological interventions [5,6]. In addition, Stone et al. demonstrated a histopathologic analysis of skin injuries from fluoroscopy [7]. However, to our knowledge, there are few studies related to radiation-induced ulcer in Korea. Thus, we aimed to present several cases of radiation-induced ulcers in our clinics, and to investigate the characteristics of radiation-induced ulcers. \\ Original Article \\ Received: July 22, 2018 \\ Revised: August 30, 2018 \\ Accepted: August 31, 2018

\section{Corresponding author: \\ Sik Namgoong, M.D.} \\ Department of Plastic Surgery, Korea University \\ Guro Hospital, 148 Gurodong-ro, Guro-gu, \\ Seoul 08308, Korea \\ Tel: +82-2-2626-3334 \\ Fax: +82-2-868-7484 \\ E-mail: siknamgoong@gmail.com
}

This is an Open Access article distributed under the terms of the Creative Commons Attribution Non-Commercial License (http://creativecommons.org/licenses/by-nc/4.0/) which permits unrestricted non-commercial use, distribution, and reproduction in any medium, provided the original work is properly cited.

(C) 2018 Korean Wound Management Society 


\section{Methods}

Data from the maintained databases at our center were retrospectively reviewed. All the patients who visited for the treatment of radiation-induced ulcers between 2010 and 2015 were included in the analysis. We reviewed medical charts and operative data, including patient demographics, photographs, surgical techniques, and postoperative outcomes. If necessary, tissue cultures were taken during surgical debridement in the operating room, and microbiological analysis was performed. Based on the wound culture results, systemic antibiotics were used in cases where slough was not eliminated despite appropriate debridements. Postoperative complications, including hematoma, seroma, necrosis, and skin graft failure, were assessed. On discharge from the hospital, patients returned for regular follow-up appointments in the outpatient clinic. The follow-up appointments were scheduled with short intervals in the first 4 weeks after discharge. After wound healing was complete and if no complication was observed, further followup was conducted at 2, 6, and 12 months post-operation.

\section{Results}

In total, five patients were included in the analysis and administered intravenous antibiotic treatment; three patients underwent local flap and one patient underwent split-thickness skin graft. Also, one patient underwent both local flap and splitthickness skin graft, and the other underwent allogenic keratinocyte transplantation (Tables 1,2). All the wounds were covered without incidence of severe complications, such as total flap necrosis or skin graft failure. Minor complications (wound dehiscence, partial flap necrosis) occurred in one pa-

Table 1. Demographic data of patients with radiation-induced back ulcer

\begin{tabular}{lllcl}
\hline Patient no. & Sex, Age & Procedure & $\begin{array}{c}\text { Location of } \\
\text { skin lesion* }\end{array}$ & \multicolumn{1}{c}{ Treatment** } \\
\hline 1 & M, 76 & RFA, RTx & Back & Local flap \\
2 & M, 71 & RFA, TACE & Back & Local flap \\
3 & M, 55 & RFA, TACE & Back & Local flap, STSG \\
4 & M, 86 & RFA, TACE & Back & Local flap \\
5 & F,73 & RFA & Back & Allogenic keratinocyte \\
& & & & transplantation \\
\hline
\end{tabular}

RFA, radiofrequency ablation; RTx, radiotherapy; TACE, transarterial chemoembolization; STSG, split-thickness skin graft.

*Each patient had only one lesion, **The treatment included intravenous antibiotics, surgical debridement, and conventional dressing. tient, which healed after the split-thickness skin graft procedure. At a mean follow-up of 11 months, all patients were healed completely without any recurrence.

\section{Case presentations}

Case 1

A 71-year-old male patient was referred to our plastic surgery clinic from the department of dermatology for the treatment of a large, dirty ulcer on his back that had developed five months ago. The patient had a history of having undergone radiofrequency ablation and transarterial chemoembolization treatment for hepatocellular carcinoma eight months ago. With intravenous antibiotic treatment, he underwent a total of four surgical debridement procedure with local flaps in total (Fig. 1).

\section{Case 2}

The patient was a 76-year-old man with a large, painful ulcer that had appeared on his back seven months ago. The size of the ulcer was $5 \mathrm{~cm} \times 4.5 \mathrm{~cm}$. The patient had undergone radiofrequency ablation and radiotherapy treatment for hepatocellular carcinoma two years ago. His wound was treated with lumbar artery perforator pedicled V-Y advancement flap coverage with intravenous antibiotic treatment (Fig. 2).

\section{Case 3}

A 55-year-old male patient was referred to our department from the department of dermatology for his back ulcer. The ulcer occurred a year ago and had been intractable to conventional dressing during the year. The size of the ulcer was $5 \mathrm{~cm}$

Table 2. Microorganisms isolated from wound

\begin{tabular}{|c|c|c|c|c|}
\hline Patient no. & Sex, Age & Bacteria & $\begin{array}{l}\text { Treatment } \\
\text { antibiotic }\end{array}$ & $\begin{array}{c}\text { Antibiotic } \\
\text { treatment } \\
\text { duration (day) }\end{array}$ \\
\hline 1 & $M, 76$ & $\begin{array}{l}\text { Klebsiella } \\
\text { pneumonia }\end{array}$ & $\begin{array}{l}\text { Cefminox, } \\
\text { Levofloxacin }\end{array}$ & 76 \\
\hline 2 & $\mathrm{M}, 71$ & $\begin{array}{l}\text { Enterococcus } \\
\text { faecalis }\end{array}$ & Ciprofloxacin & 34 \\
\hline 3 & $M, 55$ & $\begin{array}{c}\text { Acinetobacter } \\
\text { baumannii }\end{array}$ & $\begin{array}{l}\text { Tigecycline, } \\
\text { Colistin, Vancomycin }\end{array}$ & 95 \\
\hline 4 & $M, 86$ & MRSA & $\begin{array}{l}\text { Levofloxacin, } \\
\text { Vancomycin }\end{array}$ & 41 \\
\hline 5 & $\mathrm{~F}, 73$ & $\begin{array}{l}\text { Enterobacter } \\
\quad \text { kobei, Klebsiella } \\
\text { oxytoca }\end{array}$ & Ciprofloxacin & 8 \\
\hline
\end{tabular}

MRSA, methicillin-resistant Staphylococcus aureus. 

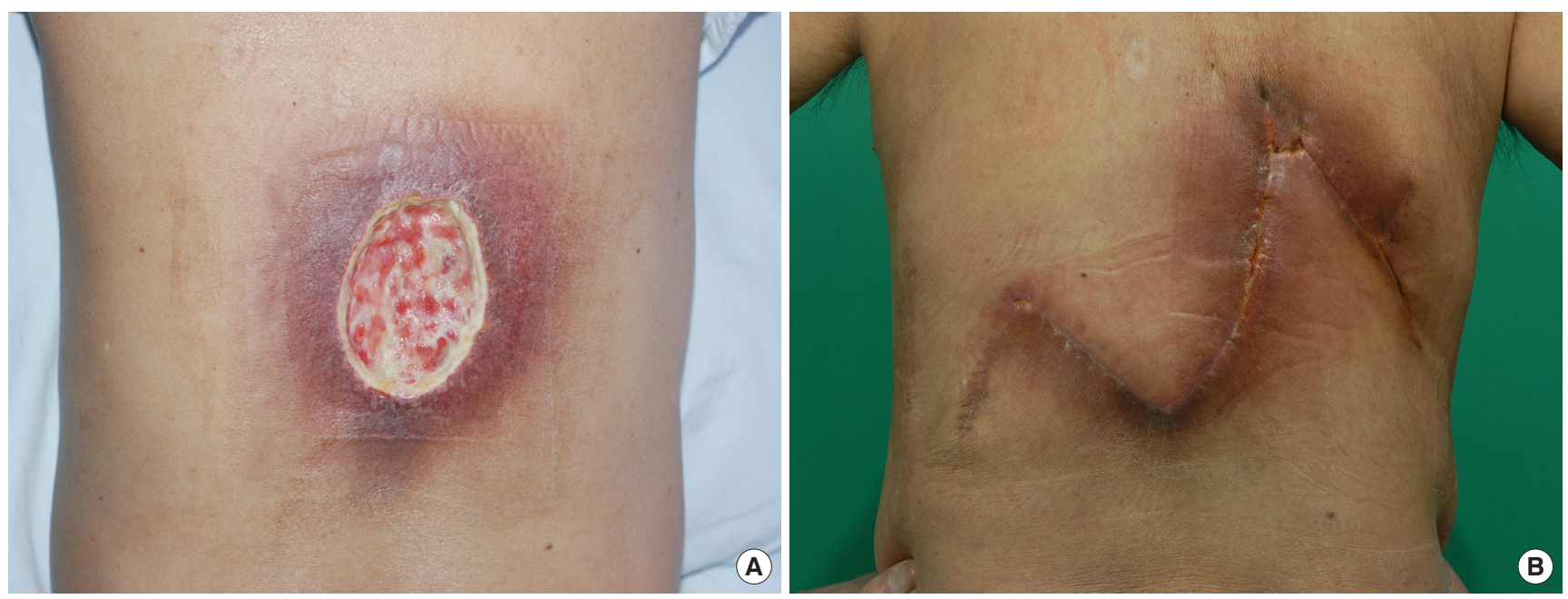

Fig. 1. A case treated with a local flap. (A) Preoperative photograph; large, dirty ulcer on the back. (B) Clinical photograph at the outpatient clinic after discharge (36 days post-operation).
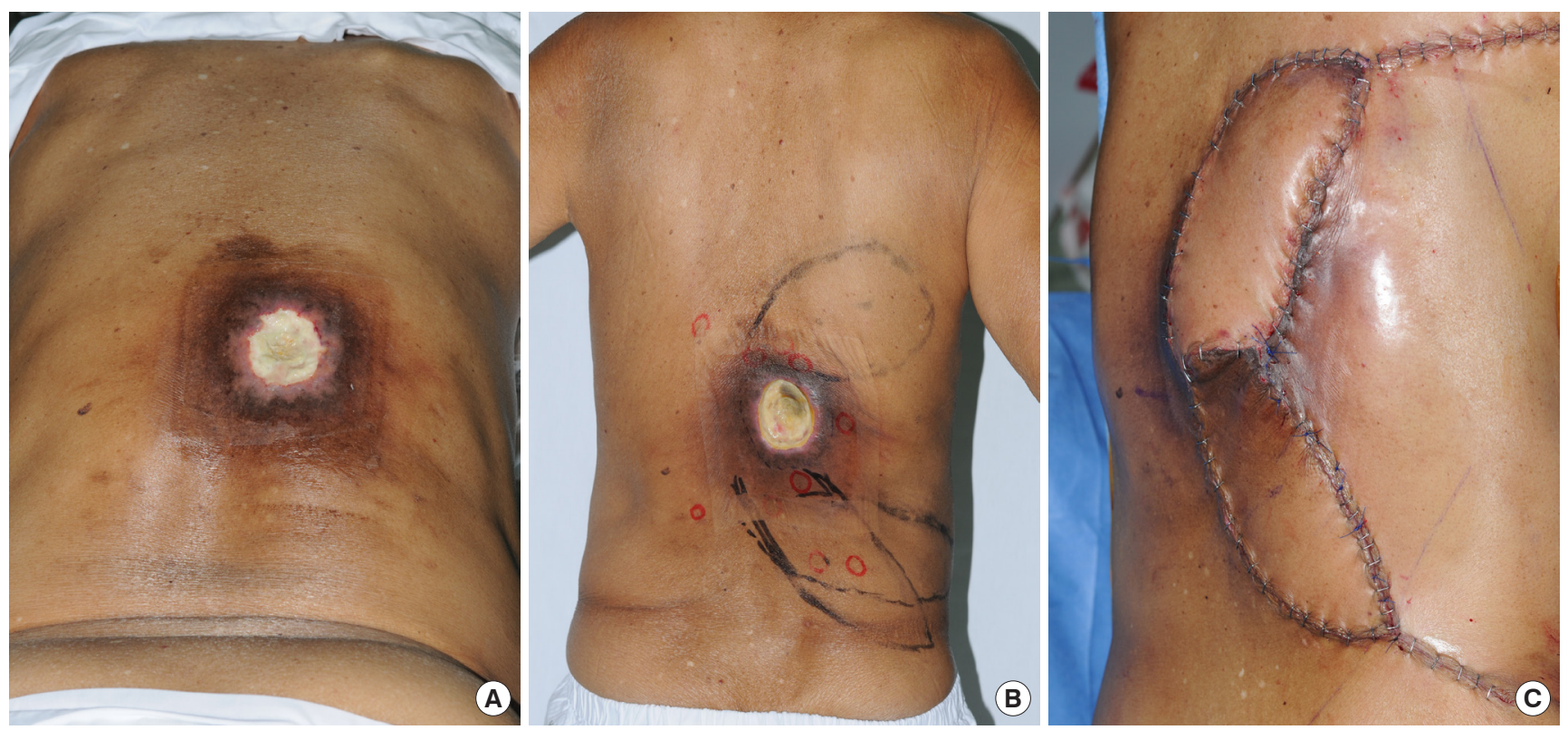

Fig. 2. A photograph of perforator-based local flap. (A) Preoperative photograph; the size of the ulcer was measured as $5 \mathrm{~cm} \times 4.5 \mathrm{~cm}$. (B) Preoperative markings were drawn including lumbar artery perforator pedicled V-Y advancement flap. (C) Immediate postoperative photo.

$\times 5 \mathrm{~cm}$. The patient had a history of having undergone radiofrequency ablation and transarterial chemoembolization treatment for hepatocellular carcinoma 1.5 years ago. He underwent several surgical debridement procedures with local flap, along with intravenous antibiotic treatment. However, partial skin necrosis and wound dehiscence had occurred. After additional surgical debridement procedures, the wound was covered with a split-thickness skin graft (Fig. 3).
Case 4

A 73-year-old woman presented with a 2-week history of a necrotic skin lesion on her back area. She underwent radiofrequency ablation 3 weeks ago as treatment for persistent atrial fibrillation. The size of the lesion was $4 \mathrm{~cm} \times 3 \mathrm{~cm}$. She had undergone debridement procedures and allogenic keratinocyte transplantation, along with intravenous antibiotic treatment. At the 3-month follow-up visit, local recurrence of ulcer was not seen (Fig. 4). 

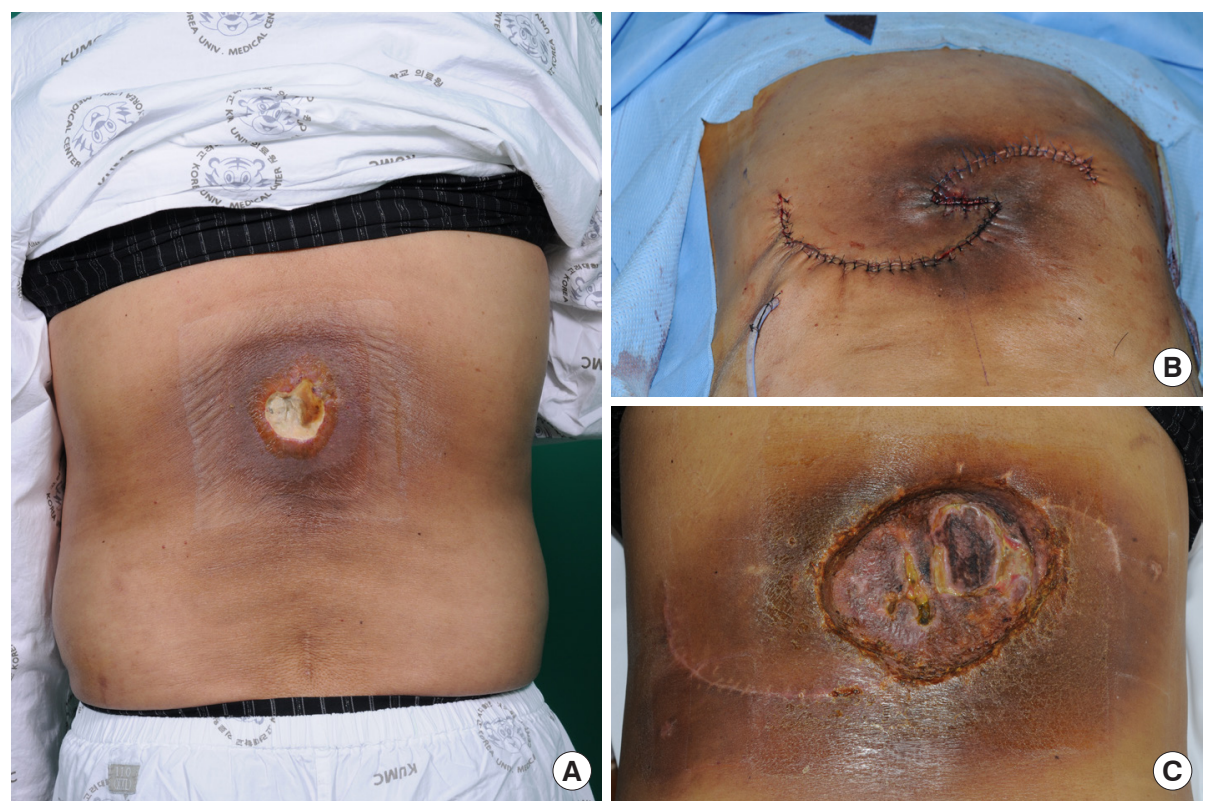

Fig. 3. The wound covered with skin graft. (A) Preoperative photograph; the size of the ulcer was measured as 5 $\mathrm{cm} \times 5 \mathrm{~cm}$. (B) Immediate postoperative photo after debridement with local flap. (C) Follow-up photograph after covering with split-thickness skin graft (three weeks post-operation).
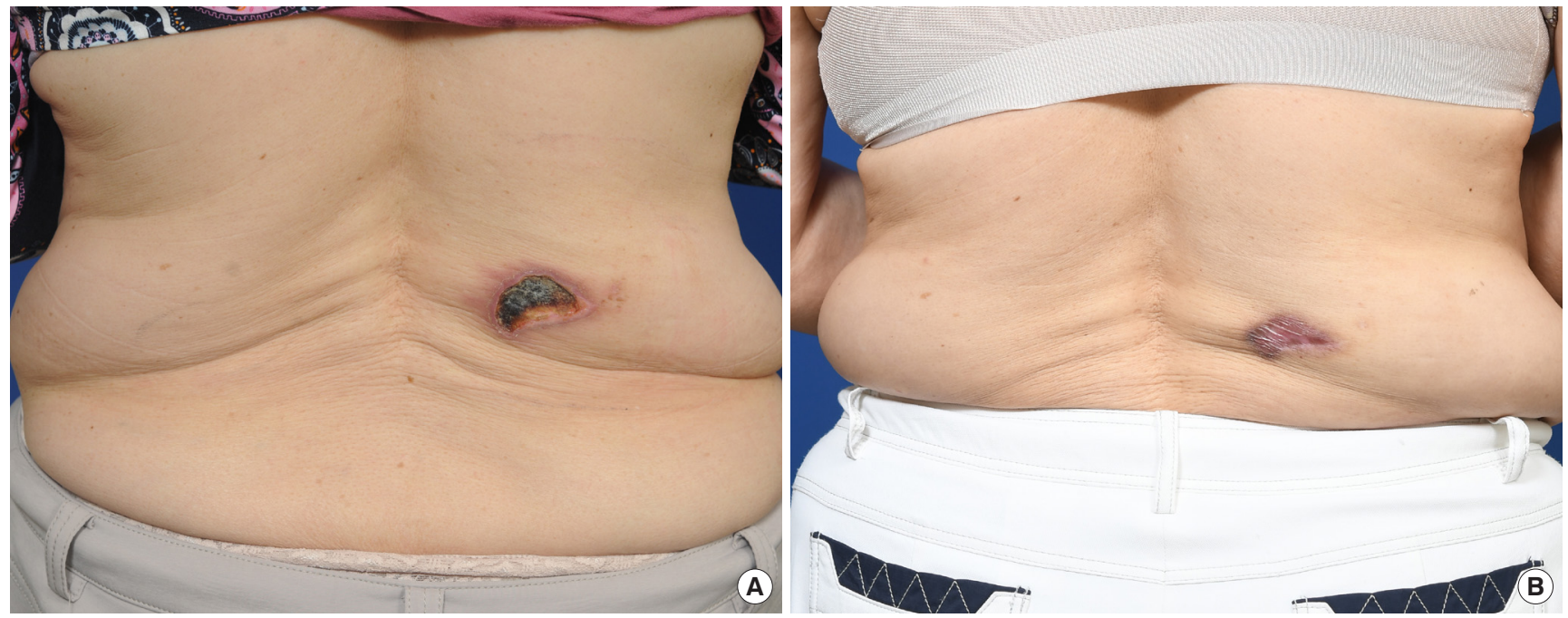

Fig. 4. A case treated with allogenic keratinocyte transplantation. (A) Initial photograph at the outpatient clinic. (B) Follow-up photograph after 3 months.

\section{Discussion}

The use of fluoroscopic interventions is now increasing because these are less invasive than conventional procedures. Numerous less invasive fluoroscopically-guided procedures have provided great benefits with fewer complications for many patients [8]. Given that fluoroscopic procedures have been more commonly used, radiation-related complications should be paid attention to. Several reports warned of the risks related to radiation-related skin injuries following radiological interventions $[5,6]$. However, there are few studies related to this in Korea.

Radiation-related skin lesions are dose-dependent and tend to occur when the patient's skin is exposed to high doses of Xrays. The site with the highest risk is the skin area where the $\mathrm{X}$ ray beam penetrates the patient's body due to the relatively poor penetrability of X-rays. The concept of absorbed dose can be used to measure the amount of radiation to which the body tissue has been exposed. Radiation dose thresholds have been established for different skin changes, varying from erythema of the skin to deep ulcers $[9,10]$. However, these threshold doses vary depending on patient susceptibilities and the radia- 
tion exposure site [7]. For example, Wagner et al. warned that patients with connective tissue disease and diabetes mellitus would be at an elevated risk of adverse skin complications [11].

There is not much difference in treatment from other ulcers, despite the complex pathophysiology of radiation-induced ulcers. A skin graft or a local musculocutaneous flap is used for the treatment of large, deep back ulcers $[10,12]$. Barnea et al. suggested that surgery is the preferred option, as the ulcer lesion, even if not malignant, is intractable to conventional dressing treatment. When surgery is performed, the surgeon should include the surgical debridement of the ulcer lesion at healthy-looking margins and treatment with a skin graft or a local flap, in light of the knowledge that the healing process may be slower and more complex in radiation-exposed skin than in normal skin [12].

Although ulcer diagnosis and treatment are important, prevention of ulcers takes priority. During cardiac and hepatic fluoroscopic procedures, radiation exposure can vary based on the anatomic location, and the maximum radiation dose is delivered to the ninth thoracic vertebra [13]. In addition, different regions of the body exhibit various grades of radiosensitivity. For example, the back is more sensitive than the neck [14]. During cardiac and hepatic fluoroscopic procedures, the back receives the highest $\mathrm{X}$-ray dose and is especially sensitive; thus, radiation-induced ulcers are more common on the back than other sites. Thus, every physician and radiologist should make an effort to reduce the fluoroscopic procedure time and to limit excessive exposure at each projection [15]. The single most important factor for radiation-induced ulcers is the exposure time at a single site. Physicians may reduce radiation-related skin injuries by undergoing training in the technical performance of procedures. Moreover, to reduce procedure time, the fluoroscopy time should be monitored to ensure the exposure time is kept to a minimum and within the standard limit of a fluoroscopic procedure [9]. Additionally, setting up a small irradiation target site to reduce the skin area exposed to radiation is important to prevent radiation-induced ulcers [16].

For the primary care physicians with whom such patients have initially met, attention must be given to the history of radiation exposure for patients with chronic back ulcers. Radiation-induction ulcers tend to be painful, intractable with conventional dressings, and frequently occur on the back area. These clinical characteristics are key factors in making a diagnosis. Physicians are often uninterested in previous procedure history, and additional information must therefore be obtained from medical charts when a radiation-induced ulcer is clinically suspected [1].

Patients who have undergone fluoroscopic procedures may be at an elevated risk of ulcer formation, because the previous radiation exposure has lowered their radiation threshold [10]. The skin conditions of patients exposed to chronic radiation should be checked regularly because radiation-induced skin damage can develop even years later [17].

Informing the patient that delayed skin changes might occur will help them understand these adverse skin reactions and identify them if they occur. Much of the anxiety of a patient with back ulcers occurs due to the strange characteristics of radiation ulcers and the lack of medical personnel's full explanations [10].

This study has limitations. The number of patients with radiation-induced ulcers might have been considerably underreported. When they came to our clinic, we did not know why the lesion had appeared if they did not notify us of previous radiation exposure. Therefore, the actual cases will be much higher in number than the current number of patients enrolled in our study. In addition, treatment of ulcer lesions is important for wound healing as well as long-term stability. The radiation-exposed skin might be susceptible to recurrence of the ulcer lesion even after wound healing occurs. However, all our patients, except for one, had been treated for hepatocellular carcinoma and undergone radiofrequency ablation and radiotherapy. They had difficulty making follow-up visits to the outpatient clinic owing to their poor general condition. Although their skin lesions healed, they died of terminal-stage cancer; thus, we could not take any clinical photographs at the long-term follow-up. Moreover, we could not analyze the long-term stability of the radiation-induced ulcer treatment. Thus, the long-term stability of radiation-induced ulcer treatment should be analyzed in further studies with a larger number of enrolled patients.

As fluoroscopic interventions are gaining more popularity due to being less invasive, more efforts are needed to reduce adverse skin reactions in patients who undergo such treatment. Lee et al. suggested that this necessitates multidisciplinary approaches to diagnose and treat patients, including interventional, dermatologic procedures, and plastic surgery [18]. We would like to emphasize that the present article is the first to demonstrate radiation-induced ulcers in Korea; and our findings may further help the study of radiation-induced ulcers and establish the importance of taking a multidisciplinary approach when caring for such patients. 


\section{Conclusion}

Radiation-induced back ulcers are easily overlooked. Thus, accurate assessment and multidisplinary approaches for the treatment of such injuries are crucial.

\section{Conflicts of interest}

No potential conflict of interest relevant to this article was reported.

\section{References}

1. Frazier TH, Richardson JB, Fabré VC, et al. Fluoroscopy-induced chronic radiation skin injury: a disease perhaps often overlooked. Arch Dermatol 2007;143:637-40.

2. Dehen L, Vilmer C, Humiliere C, et al. Chronic radiodermatitis following cardiac catheterisation: a report of two cases and a brief review of the literature. Heart 1999;81:308-12.

3. Koenig TR, Wolff D, Mettler FA, et al. Skin injuries from fluoroscopically guided procedures: part 1 , characteristics of radiation injury. Am J Roentgenol 2001;177:3-11.

4. Koenig TR, Mettler FA, Wagner LK. Skin injuries from fluoroscopically guided procedures: part 2 , review of 73 cases and recommendations for minimizing dose delivered to patient. Am J Roentgenol 2001;177:13-20.

5. Wagner LK, Eifel PJ, Geise RA. Potential biological effects following high X-ray dose interventional procedures. J Vasc Interv Radiol 1994;5:71-84.

6. Dandurand M, Huet P, Guillot B. Secondary radiodermatitis caused by endovascular explorations: 5 cases. Ann Dermatol Venereol 1999;126:413-7.

7. Stone MS, Robson KJ, LeBoit PE. Subacute radiation dermatitis from fluoroscopy during coronary artery stenting: evi- dence for cytotoxic lymphocyte mediated apoptosis. J Am Acad Dermatol 1998;38:333-6.

8. Hashimoto I, Sedo H, Inatsugi K, et al. Severe radiation-induced injury after cardiac catheter ablation: a case requiring free anterolateral thigh flap and vastus lateralis muscle flap reconstruction on the upper arm. J Plast Reconstr Aesthet Surg 2008;61:704-8.

9. The Food and Drug Administration. Avoidance of serious Xray-induced skin injuries to patients during fluoroscopicallyguided procedures. Med Bull 1994;24:7-17.

10. Vlietstra RE, Wagner LK, Koenig T, et al. Radiation burns as a severe complication of fluoroscopically guided cardiological interventions. J Interv Cardiol 2004;17:131-42.

11. Wagner LK, McNeese MD, Marx MV, et al. Severe skin reactions from interventional fluoroscopy: case report and review of the literature. Radiology 1999;213:773-6.

12. Barnea Y, Amir A, Shafir R, et al. Chronic radiodermatitis injury after cardiac catheterization. Ann Plast Surg 2002;49: 668-72.

13. Calkins H, Niklason L, Sousa J, et al. Radiation exposure during radiofrequency catheter ablation of accessory atrioventricular connections. Circulation 1991;84:2376-82.

14. Grosch D. Biological effects of radiations: Elsevier; 2012.

15. Nahass GT. Acute radiodermatitis after radiofrequency catheter ablation. J Am Acad Dermatol 1997;36:881-4.

16. Miralbell R, Maillet $P$, Crompton NE, et al. Skin radionecrosis after percutaneous transluminal coronary angioplasty: dosimetric and biological assessment. J Vasc Interv Radiol 1999; 10:1190-4.

17. Nahass GT, Cornelius L. Fluoroscopy-induced radiodermatitis after transjugular intrahepatic portosystemic shunt. Am J Gastroenterol 1998;93:1546-49.

18. Lee J, Hoss D, Phillips TJ. Fluoroscopy induced skin necrosis. Arch Dermatol 2003;139:140-2. 